\title{
Unintentional Exposure and Incidental Findings during Conventional Chest Radiography in the Pediatric Intensive Care Unit
}

\author{
Mohamad-Hani Temsah \\ King Saud Medical City \\ Ayman Al-Eyadhy \\ King Saud Medical City \\ Fahad Alsohime ( $\square$ dr.fahad.alsohime@gmail.com ) \\ King Saud Medical City \\ Saeed Majed Nassar \\ King Saud Medical City \\ Talal Nabil AlHoshan \\ King Saud Medical City \\ Hatim Abdullah Alebdi \\ King Saud Medical City \\ Faisal Almojel \\ King Saud Medical City \\ Muath Abdullah AlBattah \\ King Saud Medical City \\ Omendra Narayan \\ university of manchester \\ Gamal Mohamad Hasan \\ assiut \\ Abdullah Abujamea \\ King Saud Medical City
}

Research article

Keywords: chest x-ray, critical care, cropping, incidental findings, overexposure, PIM2

Posted Date: September 19th, 2019

DOI: https://doi.org/10.21203/rs.2.14660/v1 
License: (c) (i) This work is licensed under a Creative Commons Attribution 4.0 International License. Read Full License

Version of Record: A version of this preprint was published at Medicine on March 5th, 2021. See the published version at https://doi.org/10.1097/MD.0000000000024760. 


\section{Abstract}

Background: Anatomical overexposure is common in CXRs of pediatric patients. However, overexposure may reveal incidental findings that can help to guide patient management or warrant quality improvement.

Objective: To assess the prevalence of overexposure in chest X-ray (CXRs) in pediatric intensive care unit (PICU); and identify the incidental findings within overexposed areas.

Materials and methods: Retrospective cohort study of children admitted to PICU. Two independent evaluators reviewed patient's charts and digital CXRs according to the American College of Radiology standards; to evaluate overexposure of the anatomical parameters and incidental findings.

Results: A total of 400 CXRs of 85 patients were reviewed. The mean number of CXRs per patient was 4.7. Almost all (99.75\%) CXRs met the criteria for overexposure, with the most common being upper abdomen (99.2\%), upper limbs (97\%) and neck (95.7\%). In addition, $43 \%$ of these X-rays were cropped by the radiology technician to appear within the requested perimeter. There was a significant association between field cropping and overexposure (t-test: $t=9.8, p<.001)$. Incidental findings were seen in $41.5 \%$ of the radiographs; with the most common being gaseous abdominal distension (73.1\%), low-positioned nasogastric tube $(24.6 \%)$, and constipation (10.3\%).

Conclusions: Anatomical overexposure in routine CXRs remains high and raises a concern in PICU practice. Appropriate collimation of the X-ray beam, rather than electronically cropping the image, is highly recommended to minimize hiding incidental findings in the cropped-out areas. Redefining the anatomic boundaries of CXR in critically ill infants and children may need further studies and consideration. Quality improvement initiatives to minimize radiation overexposure in PICU are recommended.

\section{Background}

Pediatric and neonatal patients pose an immense burden in the intensive care unit (ICU) setting; most of their mortalities correlate with birth complications and respiratory illnesses. On a global survey of 5.900 .000 deaths in the ICU for patients under the age of 5 (U5 MR), 920.000 (15.6\%) died of pneumonia, making it the second leading cause of death at the ICU in this age group (1). In another epidemiological study, it was found that the most common diagnoses at the pediatric intensive care unit (PICU) were respiratory disorders (33.26\%) (2). Of these disorders, bronchopneumonia, respiratory failure, and acute bronchitis were the most commonly encountered. At a regional level, the highest mortality due to lower respiratory infection were among children under 5 years old (3). Other respiratory illnesses, such as acute asthma exacerbations, were reported to have increasing rate of PICU admission (4).

With respiratory disorders being a leading concern in the pediatric and neonatal ICUs, a large number of chest $\mathrm{x}$-rays (CXRs) are ordered daily for diagnostic and progress assessment. Moreover, the 
management of these critically ill children often includes various modalities that can potentially act as risk factors leading to the development or worsening of respiratory diseases, thereby increasing the number of CXRs orders. These modalities may include fluids and nutrition through intravascular catheters (5), and respiratory support with mechanical ventilation. These interventions might necessitate further radiological studies which in turn can potentially produce a higher risk of radiation exposure, especially if not done within the recommended portable CXRs parameters.

Unfortunately, anatomical overexposure is very common in CXRs of pediatric and neonatal patients with up to $85 \%$ of CXRs including the whole abdomen (6). On the other hand, overexposure may reveal incidental findings that can further help in guiding patient management or warrant quality improvement $(7-8)$.

The current study aims to quantify and determine the anatomical regions that were excessively exposed to radiation in CXRs done for children in the PICU at a tertiary care hospital. In addition, the study evaluates possible patients' factors that are associated with radiation overexposure, along with their associated incidental radiological findings, especially in the electronically cropped out areas.

\section{Methods}

A retrospective observational study was performed at the Pediatric Intensive Care Unit (PICU) in a tertiary teaching hospital in Riyadh, Saudi Arabia. A total of 684 patients were admitted to the PICU during the study period from January 2015 to June 2016. The patient medical record numbers (MRNs) were obtained from the PICU database registry. The inclusion criteria were: children admitted to the PICU during the study period surviving until discharge; however, we excluded repeated admissions. The population was stratified by age, length of stay, and gender; then a sample of 85 patients was selected by a simple random method. The total number of chest x-rays (CXRs) for these patients was 400 . These CXRs were reviewed independently by two members of the research team to assess the extent of the exposure field and presence of any incidental findings (6). If the two reviewers had any discrepancy in their assessment, a third assessment was performed by a dedicated PICU physician for confirmation. The CXRs were performed for various medical indications (such as pneumonia, bronchiolitis, etc.) by on-call radiographers, using portable X-ray machine (Siemens Mobilett Mira Max) available in the PICU around the clock. The chest radiographs were viewed using the Picture Archiving and Communication System (PACS) which is accessible by physicians on the hospital intranet.

The radiographs were evaluated for unnecessary anatomical exposure based on the recommended guidelines of the American College of Radiology (ACR) (9-10). An ideal chest radiograph should extend vertically from the first ribs ( $\mathrm{T} 1)$ at the apices of the lung to the costophrenic angles and horizontally including the lateral margins of the ribs (Figure 1a). Overexposure was determined by an extension of 2 $\mathrm{cm}$ or more beyond these recommended boundaries (6). The defined regions for excessive exposure that were accounted for in this study were: the head (mandibular prominence and upwards), neck (cervical spine above the first rib), upper limbs (head of the humerus), upper abdomen (beneath the diaphragms), 
lower abdomen (Pelvis), and lower limbs (head of the femur). We also accounted for the post-exposure adjustment to the radiographs, mainly electronic collimation (otherwise known as field cropping) (1112). This form of collimation is done by $x$-ray technicians to confine the visible area in the radiograph as requested by the physician, thus excluding the extraneous parts of the x-ray image (Figure 1b).

Incidental findings were accounted for in the areas apart from the suggested boundaries of the chest radiograph according to the ACR. The targeted incidental findings were: a misplaced low nasogastric tube, excessive intraluminal abdominal gases, constipation features, and hand of the caregiver (attending nurse). A high nasogastric tube (NGT) was defined by finding its tip at or above the lower esophageal sphincter, whereas a low NGT was defined by finding its tip on the floor of the stomach (creating a coiled appearance in some instances) or going into the duodenum. As for abdominal gases, the intraluminal gases that appear primarily as dilation and opacification were checked (13) as this can denote numerous causes such as bowel obstruction, constipation, etc. Radiologist/attending physician reports were graded based on their availability with every radiograph.

Data was collected using a paper-based data abstraction sheet. Two independent evaluators assessed the same sample. The collected data was revised by a physician to settle any differences between the two data sets. Data were analyzed using the commercially available analytical program SPSS, Version 21. Means \pm Standard deviation, frequencies and percentages were tabulated. The X-ray over-exposures, incidental findings, and data were nested within each of the 85 subjects. The number of CXRs were variable for each child. We reviewed all CXRs taken on each child throughout the admission time. As such, these over-exposures and incidental findings were entered as multiple response dichotomy variables. We then used the multiple response dichotomy analysis methods to describe these findings. Therefore, their total percentage might not add to a $100 \%$ because each child could have a combination of one or more exposures or incidental findings at the same time.

The Sum function was used to compute the total over-exposed organs and the total incidental findings for each X-ray reviewed for each child by adding them up, and the resulting variables were total exposures and incidental findings.

The independent group's t-test was used to assess the statistical differences in mean over-exposed organs and the mean incidental findings across dichotomized levels of the CXR field crop (Yes/ No), and the difference was expressed in terms of a measure of effect size statistic (Cohen's $d$ ).

Data was then restructured into admissions by utilizing the transpose feature in the analytical program, where each child admission had their means of total exposures across admission and means of incidental findings tabulated into rows, while the children's basic characteristics like age, gender, body mass index, and length of stay, remained the same.

To further understand the joint effects of the child and their admission characteristics on the mean number of over-exposed body parts per child (per admission), a Multivariate Linear Regression model was used for analysis. Data were tabulated in a structure that reflects the child-admission as a unit of 
analysis and the mean number of over-exposed organs per admission was set as an outcome variable in a sub-set dataset. In general, we had at least 11.9 subjects for each predictor; as such, we decided to use the least but most relevant predictors in the model to attain parsimony in the Multivariate model.

\section{Results}

A total of 85 patients were included in this study. The demographic characteristics of the study subjects are presented in Table 1. Approximately 400 CXRs were taken during their PICU stay. A total of 399 out of the $400(99.75 \%)$ reviewed chest radiographs were overexposed with only one patient without anatomical over exposure (0.025\%). Figure $1 \mathrm{c}$ illustrates such anatomical overexposure, that includes the abdomen, neck and upper limbs, and revealing abdominal gaseous distention and low nasogastric tube. Around 43.2\% CXRs were cropped while $56.8 \%$ were not. Figures $1 \mathrm{a}$ and $1 \mathrm{~b}$, mentioned earlier, illustrate an example of chest X-ray with cropped boundaries. The mean number of CXRs per patient was 4.7 (ranging from 1 to 36 ). The predominant primary diagnoses were respiratory disorders (51.8\%), most of which were respiratory failure, bronchiolitis, and respiratory syncytial virus infections. Other dominant admissions that required CXRs included, but not limited to, patients admitted for post-surgical care and patients with seizures (Table 2).

The majority of CXRs exposures exceeded the boundaries as per international recommendations (Figure 2). The frequency of unnecessary regions found in the CXRs was upper abdomen (99.2\%), upper limbs (97\%), neck (95.7\%), head (58.6\%), lower abdomen/pelvis (44.4\%) and lower limbs (28.8\%). In addition, many $\mathrm{x}$-rays images (43\%) were cropped by the radiology technician to appear within the requested perimeter. There was a significant association between field cropping and overexposure (t-test: $t=9.8$, $p<.001$ ) with a large effect size (Cohen's $D=0.88$ ), denoting the magnitude in the difference in the mean number of over-exposed organs was statistically larger across field cropped CXRs. However, the association of field cropping to the number of the incidental finding was insignificant $(t=0.62, p=$ $0.538)$.

Out of the reviewed 400 radiographs, $166(41.5 \%)$ had at least one or more incidental findings (Figure 3). The most common incidental findings were increased intraluminal abdominal gases (75.3\%). Low positioned NGTs were found in $25.3 \%$, constipation in $10.3 \%$, and hand of the caregiver was found in $6 \%$. High positioned NGT was also detected in $4.2 \%$ of the CXRs. None of these incidental findings were noted in the radiologist report when such reports were available in PACS. We did not identify other possible serious findings such as free intra-abdominal gas in the studies CXRs.

A multivariate linear regression model was conducted to examine the association between patient characteristics such as age, height, body mass index (BMI), weight...etc. and the total anatomical overexposures on average (Table 3 ). Older patients tended to have significantly less overexposure compared to the younger population ( $t$-value $=-4.73, p<0.001$ ), and children with the higher pediatric index of mortality (PIM2) (severity of illness score that is being used in PICUs upon patients admission to 
predict probability of death based on such score) also had a greater chance of anatomical overexposure $(\mathrm{t}$-value $=1.99, \mathrm{p}=0.049)$.

\section{Discussion}

Repeated CXRs is a common requirement for monitoring critically ill children admitted to the PICU. However, anatomical overexposure is a health concern that needs to be addressed through quality improvements due to the potential long term risks of radiation over-exposure especially in pediatric age groups. The current study findings indicated that children admitted to the PICU were overexposed to Xrays during diagnostic imaging. These results are in agreement with those of Bader et al. (6). who indicated that patients admitted in the Neonatal Intensive Care Unit (NICU) had shown a level of exposure excessive to that of international recommendations by including irrelevant organs when performing chest and abdominal radiographs. While the current definition of CXRs boundaries may have led to overestimation of radiological exposure, overexposure remains high (6). The anatomical over-exposure detected in the current study might result in an increase in the total radiation dose the patient received and its impact on the patient health cannot be identified on the short term outcome. Moreover, the actual impact of radiation over-exposure was not within the scope of the current study. However, further intervention such as educational campaigns for radiology technicians to improve their performance and to enhance beam dose collimation that can result in minimizing anatomical over-exposure and consequently total dose radiation might be an urgent need for this setting. This is especially important in the younger children and those with higher PIM2 severity scores upon PICU admissions. Another possible area of improvement that might be considered is to redefine the anatomic boundaries of CXR, to include lower neck and upper abdomen, which may trigger further discussion and necessitate further studies and consideration in acute care setting such as NICU or PICU. However, if such practice allowed, the extent of the total dose of radiation and the expected benefits in the patient's management and short term outcome must be weighed against the possible risk of long term consequences of radiation.

The current study showed that $29.5 \%$ of the inserted NGTs were mis-positioned (high or low) which is consistent with the findings of Sivit et al. (7). Also, the current study found that $6 \%$ of CXRs showed extraneous adult finger. This finding signals another area warranting quality improvement initiatives related to patient imaged field similar to the work by Tynan et al. to reduce rate of appearance of extraneous adult finger which was reported in $15 \%$ of films in their study (8).

Electronic collimation, also known as "field cropping", was a significant factor in our study, although it was apparently made as a facilitator for the workflow of the radiographer and the radiologist. It simplified the process of imaging and interpretation of the primary indication on the radiograph (10). However, by masking additional areas of the radiograph, it potentially acts as a deterrent when it comes to detecting incidental findings (such as misplaced NGTs or constipation) and hiding the level of anatomical exposure. In our study, chest radiographs that underwent electronic collimation were more likely to have anatomical overexposure. Nonetheless, its application did not show any association with incidental findings, as they were found similarly in either the cropped or non-cropped images. It was recently 
reported in the adult literature that improper use of electronic collimation could hide diagnostically useful details as well as hiding the actual size of the irradiated anatomy (14). This practice was proposed as a risk of concealing a systematic overexposure of patients due to improper collimation practices. When the radiation technologists in this survey were informed about the final results regarding collimation, they debated that their primary reason was to assure that the whole anatomy of interest is imaged and avoid repeating radiographs. This could also be the cause of high overexposure incidence in our study. However, electronic collimation could easily lead to over-relaxation in cropping practices, with decrease attention of the radiation technologists on the extent of irradiated anatomy.

The assessment of ionizing radiation risks is critical to maintaining the health of ICU patients that undergo many x-rays throughout their ICU stay. The Committee on Environmental Health at the American Academy of Pediatrics analyzed these risks and found that most procedures are associated with minute peril to the patient, so long as the exposure is limited to imaging necessary for medical care (15).

However, ionizing radiation used in diagnostic radiological procedures should not be under-estimated in children. It has been observed that the effects of a dose of radiation are variable depending on the given age group, elevating cancer risks with children, the most at-risk population. This is mostly attributed to the higher sensitivity of underdeveloped organs and tissue which has yet to mature, as maturity is associated with a lower risk (15-17). Accordingly, children should be exposed to the least amount of possible radiation in respect to dose and area. Many campaigns and regulations have been placed to make the radiological practice safer. A quintessential example is ALARA, an acronym meaning "As Low As Reasonably Achievable," which was put in place by the United States Nuclear Regulatory Commission. It advocates making every possible effort to maintain ionizing radiation exposure at the most reasonable level (17). The risk of overexposure associated with electronic collimation is higher where the field size was not automatically selected as per the examination protocol and with lack of intervention against oversized collimation (14).

It is essential to change the direction of radiological imaging through further implementation of safe practices. This can be demonstrated through further application of criteria such as ALARA as suggested by Rodgerson et al. (18). Implementing monthly workshops for the radiographers and nurses as a reminder of the potential hazards and to make sure to reduce the amount of exposure regarding field size and dose can be done. Another suggestion by Bader et al. is to implement a devoted team in the PICU to maintain quality control standards in terms of $x$-ray radiography. The benefits of intervention through the implementation of quality standards has been shown by Tynan et al., with a decrease in diagnosing extraneous adult fingers from $15 \%$ of the reviewed radiographs down to $5 \%(7)$.

Despite its limitation being a single center study, this is the first study to demonstrate the high prevalence of overexposures associated with CXRs in PICU that were masked by electronic cropping, and may thereafter trigger further investigations of actual PICU CXRs practices among other healthcare institutes.

\section{Conclusion}


The current definition of CXRs anatomical boundaries may have led to overestimated overexposure in the PICU. While anatomical overexposure remains alarmingly high in this acute care imaging practice, still, the optimal anatomical boundaries of CXR in critically ill infants and children need further consideration. Collimation of the X-ray beam, rather than electronically cropping the image, is highly recommended to minimize over-exposure as well as to avoid hiding incidental findings in the cropped out areas. Quality improvement initiatives to minimize overexposure and enhance patient safety in pediatric acute care are recommended, especially in younger children and those with more severe illness upon PICU admission.

\section{Abbreviations}

ACRAmerican College of Radiology

ALARAAs Low As Reasonably Achievable

BMIBody Mass Index

CXRsChest X-rays

ICUIntensive Care Unit

MRNsMedical Record Numbers

NICUNeonatal Intensive Care Unit

NGTnasogastric tube

PACSPicture Archiving and Communication System

PICUPediatric Intensive Care Unit

PIM2Pediatric Index of Mortality

U5 MRUnder 5 Mortality Rate

\section{Declarations}

Ethics approval and consent to participate:IRB Approval Ref \# 16/0373/IRB

Consent for publication: Not applicable

Availability of data and materials: Not applicable

Competing interests: There are no conflicts of interest. 
Funding: This research has been financially supported by Prince Abdullah Ben Khalid Celiac Disease Research Chair, under the Vice Deanship of Research Chairs, King Saud University, Riyadh, Kingdom of Saudi Arabia. The funding body had no role in the design of the study and collection, analysis, and interpretation of data or in writing the manuscript.

\section{Authors' contributions}

MHT, AA, FA, SMN, TNA, HAA, FA, MAA, ON, GMH, AA.

All authors contributed equally to this work.

Acknowledgements:

The authors thank Dr. Ahmed Ashri (PICU physician; CXRs cross-check) and www.hodhodata.com for their data analysis and support. This research has been financially supported by Prince Abdullah Ben Khalid Celiac Disease Research Chair, under the Vice Deanship of Research Chairs, King Saud University, Riyadh, Kingdom of Saudi Arabia.

\section{References}

1.Liu L, Oza S, Hogan D, Chu Y, Perin J, Zhu J et al. Global, regional, and national causes of under-5 mortality in 2000-15: An updated systematic analysis with implications for the sustainable development goals. Lancet. 2016;388:3027-35.

2.Lanetzki CS, de Oliveira CA, Bass LM, Abramovici S, Troster EJ. The epidemiological profile of pediatric intensive care center at hospital israelita albert einstein. Einstein (Sao Paulo, Brazil). 2012;10:16-21.

3.Burden of lower respiratory infections in the eastern mediterranean region between 1990 and 2015: Findings from the global burden of disease 2015 study. Int J Public Health. 2018;63:97-108

4.Al-Eyadhy AA, Temsah MH, Alhaboob AA, Aldubayan AK, Almousa NA, Alsharidah AM et al. Asthma changes at a pediatric intensive care unit after 10 years: Observational study. Ann Thorac Med. $2015 ; 10: 243-8$.

5.Yogaraj JS, Elward AM, Fraser VJ. Rate, risk factors, and outcomes of nosocomial primary bloodstream infection in pediatric intensive care unit patients. Pediatrics. 2002;110:481-5.

6.Bader D, Datz H, Bartal G, Juster AA, Marks K, Smolkin T et al. Unintentional exposure of neonates to conventional radiography in the neonatal intensive care units. J Perinatol. 2007;27:579-85

7.Sivit CJ, Taylor GA, Hauser GJ, Pollack MM, Bulas DI, Guion CJ et al. Efficacy of chest radiography in pediatric intensive care. AJR Am J Roentgenol. 1989;152:575-7. 
8.Tynan JR, Duncan MD, Burbridge BE. Reduction of adult fingers visualized on pediatric intensive care unit (picu) chest radiographs after radiation technologist and picu staff radiation safety education. Can Assoc Radiol J. 2009;60:182-4.

9.Radiology Aco. Acr-spr practice parameter for the performance of chest radiography (amended 2014) 2014.

10.Imura M, Tabata Y, Ishigaki R, Kuroda Y, Uranishi Y, Oshiro O. Automatic cropping method of chest radiographs based on adaptive binarization. Conference proceedings: Annual International Conference of the IEEE Engineering in Medicine and Biology Society. IEEE Engineering in Medicine and Biology Society. Annual Conference. 2013;2013:6494-7.

11.Hall EJ. Lessons we have learned from our children: Cancer risks from diagnostic radiology. Pediatr Radiol. 2002;32:700-6.

12.Bomer J, Wiersma-Deijl L, Holscher HC. Electronic collimation and radiation protection in paediatric digital radiography: Revival of the silver lining. Insights imaging. 2013;4:723-7.

13.Musson RE, Bickle I, Vijay RK. Gas patterns on plain abdominal radiographs: A pictorial review. Postgrad Med J. 2011;87:274-87.

14.Tsalafoutas IA. Electronic collimation of radiographic images: Does it comprise an overexposure risk? Br J Radiol. 2018;91(1086):20170958.

15.Risk of ionizing radiation exposure to children: A subject review. American academy of pediatrics. Committee on environmental health. Pediatrics. 1998;101:717-19.

https://www.ncbi.nlm.nih.gov/pubmed/9521965.

16.Brody AS, Frush DP, Huda W, Brent RL. Radiation risk to children from computed tomography. Pediatrics. 2007;120:677-82.

17.Comission UNR. Glossary: Alara. 2018

18.Rodgerson C. ALARA and paediatric imaging in radiation therapy: A survey of canadian paediatric imaging practice. Radiography. 2014;20:183-188.

\section{Tables}

Table 1: Subject Clinical and Demographic Characteristics. N= 85 


\begin{tabular}{|c|c|c|c|}
\hline \multicolumn{2}{|l|}{ Variables } & \multicolumn{2}{|c|}{ Frequency \% } \\
\hline \multirow[t]{2}{*}{ Age (months) } & Mean, Sd & 43.9 & \\
\hline & Range & $1-18$ & \\
\hline \multirow[t]{2}{*}{ Gender } & Female & 36 & 42.4 \\
\hline & Male & 49 & 57.6 \\
\hline \multirow[t]{2}{*}{ Height (cm) } & Mean, Sd & \multicolumn{2}{|c|}{$87.2(34.1)$} \\
\hline & Range & \multicolumn{2}{|c|}{$40-159$} \\
\hline \multirow[t]{2}{*}{ Weight (Kg) } & Mean, Sd & \multicolumn{2}{|c|}{$14.3(11.7)$} \\
\hline & Range & \multicolumn{2}{|c|}{$2-48$} \\
\hline \multirow[t]{2}{*}{ BMI } & Mean, Sd & \multicolumn{2}{|c|}{$16(3.7)$} \\
\hline & Range & \multicolumn{2}{|c|}{$10-31$} \\
\hline \multirow{2}{*}{ Length of Stay (days) } & Median(Q1,Q2) & \multicolumn{2}{|c|}{$3.5(7.4)$} \\
\hline & Range & \multicolumn{2}{|c|}{$1-38$} \\
\hline \multirow[t]{2}{*}{ Mechanical Ventilation } & Yes & 17 & 20 \\
\hline & No & 68 & 80 \\
\hline Number of Chest X-rays & $(\mathrm{n}=400)$ & & \\
\hline \multirow[t]{2}{*}{ Exposure } & Over-exposure & 399 & $99.75 \%$ \\
\hline & Under-exposure & 1 & $0.25 \%$ \\
\hline \multirow[t]{2}{*}{ Cropped } & Yes & 173 & 43.2 \\
\hline & No & 227 & 56.8 \\
\hline
\end{tabular}

Table 2: Diagnosis at Admission. 


\begin{tabular}{lcc}
\hline \hline Diagnosis at Admission & \multicolumn{2}{c}{ N=85*Percent } \\
\hline \hline Acute/ Chronic Respiratory Failure & 27 & $32.10 \%$ \\
Bronchiolitis & 19 & $22.60 \%$ \\
Post-Surgical/ O.R stay & 17 & $20.20 \%$ \\
Respiratory viral infections & 13 & $15.50 \%$ \\
Asthmatic Exacerbation & 12 & $14.30 \%$ \\
Pneumonia & 10 & $11.90 \%$ \\
Seizures, Convulsions, status epilepticus, CNS 10 & $11.90 \%$ \\
Cardiac Anomalies/Cardiac Dysfunction & 6 & $7.10 \%$ \\
DKA, Electrolyte/Acid-Base Imbalance & 6 & $7.10 \%$ \\
Septic shock & 5 & $6.00 \%$ \\
Injury, falls, and drowning/Toxicity & 4 & $4.80 \%$ \\
Acute Renal Impairment/Compromise & 3 & $3.60 \%$ \\
Hematological Disease & 3 & $3.60 \%$ \\
\hline \hline
\end{tabular}

Table 3: Multivariate Linear Regression Model explaining the joint and individual relationship between child and admission characteristics and the number of exposed organs across admission. $\mathrm{N}=85$ 


\begin{tabular}{|c|c|c|c|c|c|}
\hline & $\begin{array}{l}\text { Number of } \\
\text { organs }\end{array}$ & $\begin{array}{c}\text { exposed Standard } \\
\text { Error }\end{array}$ & $\begin{array}{l}\text { Standardized } \\
\text { Beta }\end{array}$ & $\begin{array}{l}\text { t- } \\
\text { Value }\end{array}$ & $\mathbf{P}$ \\
\hline (Constant) & 4.91 & 0.391 & & 12.572 & $<0.001$ \\
\hline Age (Months) & -0.008 & 0.002 & -0.457 & -4.726 & $<0.001$ \\
\hline PIM2 & 0.033 & 0.016 & 0.204 & 1.997 & 0.049 \\
\hline BMI & -0.034 & 0.022 & -0.148 & -1.542 & 0.127 \\
\hline Sex $=$ Male & -0.161 & 0.173 & -0.088 & -0.93 & 0.355 \\
\hline Mechanically & -0.108 & 0.184 & -0.057 & -0.59 & 0.557 \\
\hline \multicolumn{6}{|l|}{ Ventilated } \\
\hline Length Of Stay & 0.005 & 0.013 & 0.041 & 0.4 & 0.690 \\
\hline
\end{tabular}

\section{Figures}

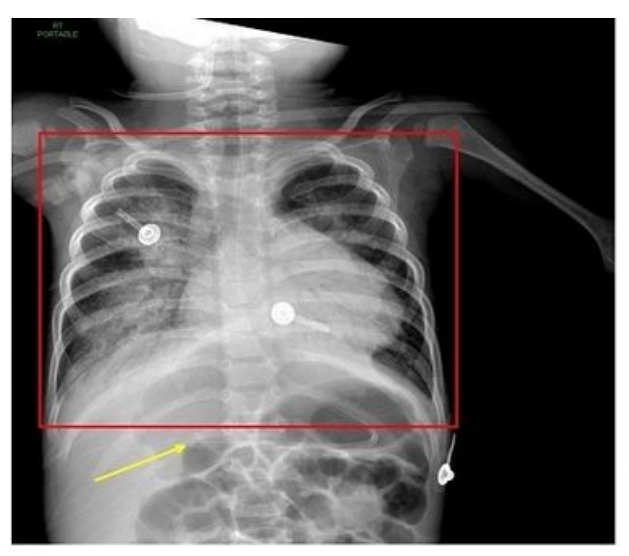

A

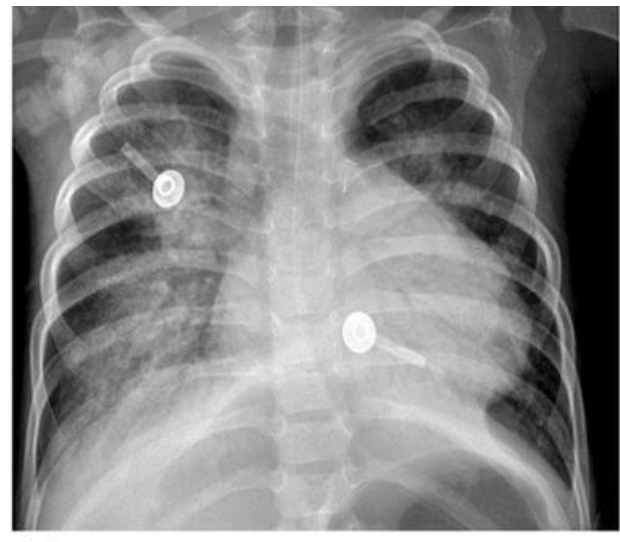

B

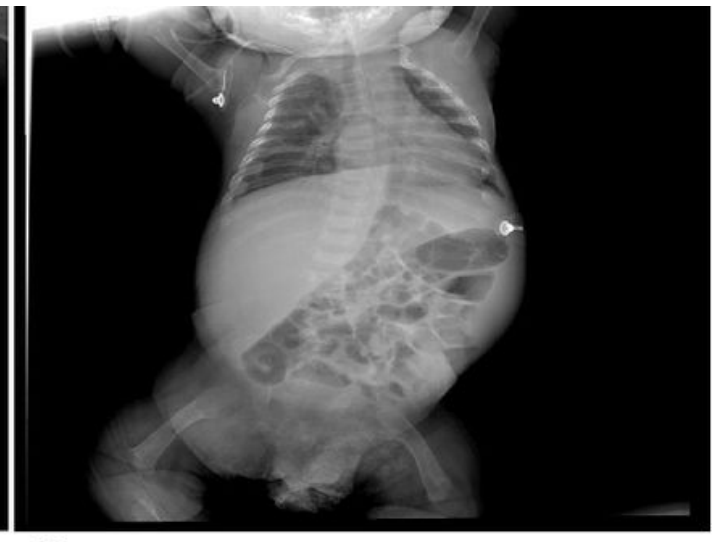

C

Figure 1

A: Red square: Recommendations for chest x-ray boundaries; Yellow Arrow: Low Nasogastric Tube. B: View after application of electronic collimation. C: Chest X-ray with anatomical overexposure. 


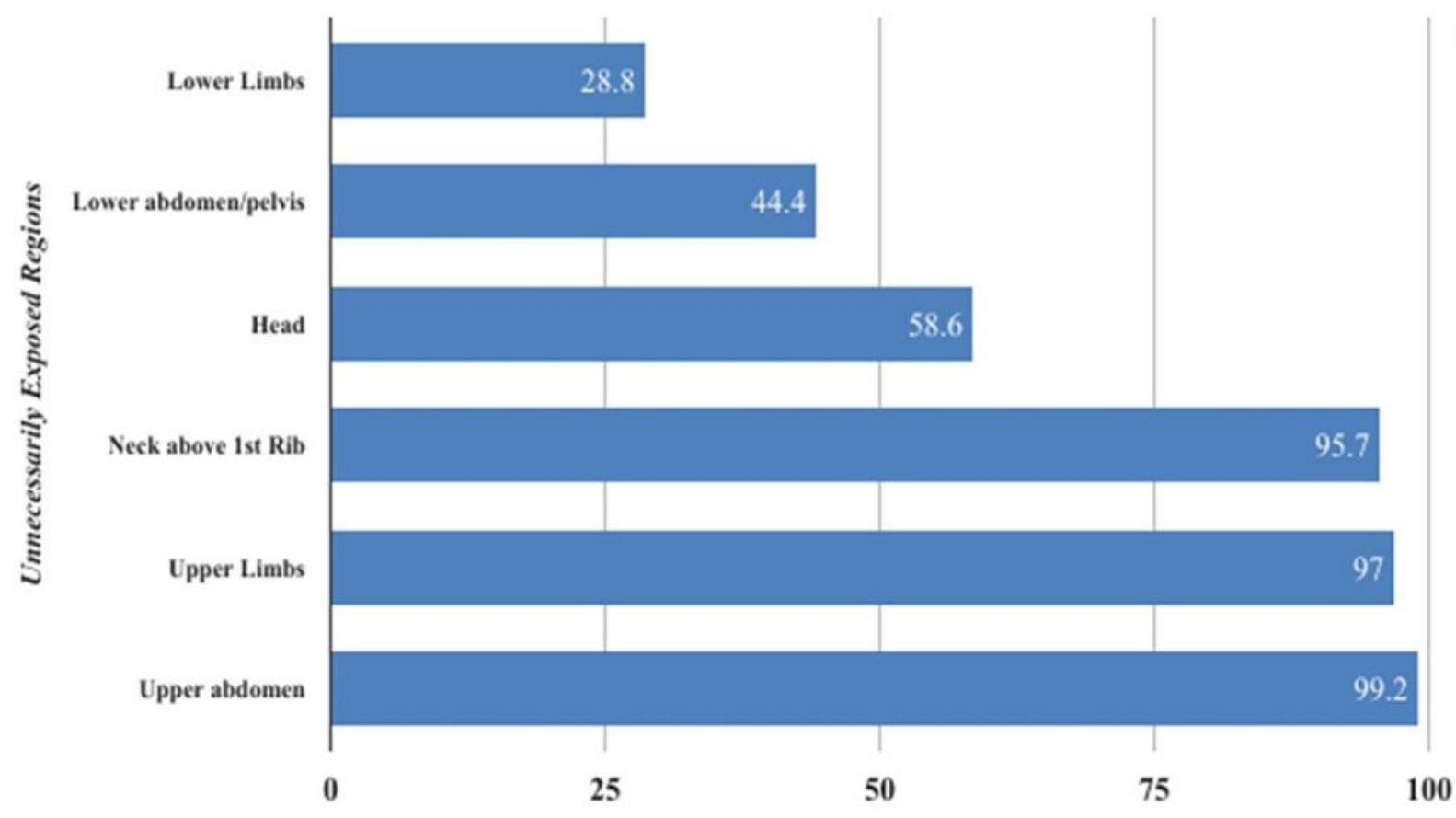

Percentage

\section{Figure 2}

Unintentional exposure of body parts per chest X-ray, N= 399

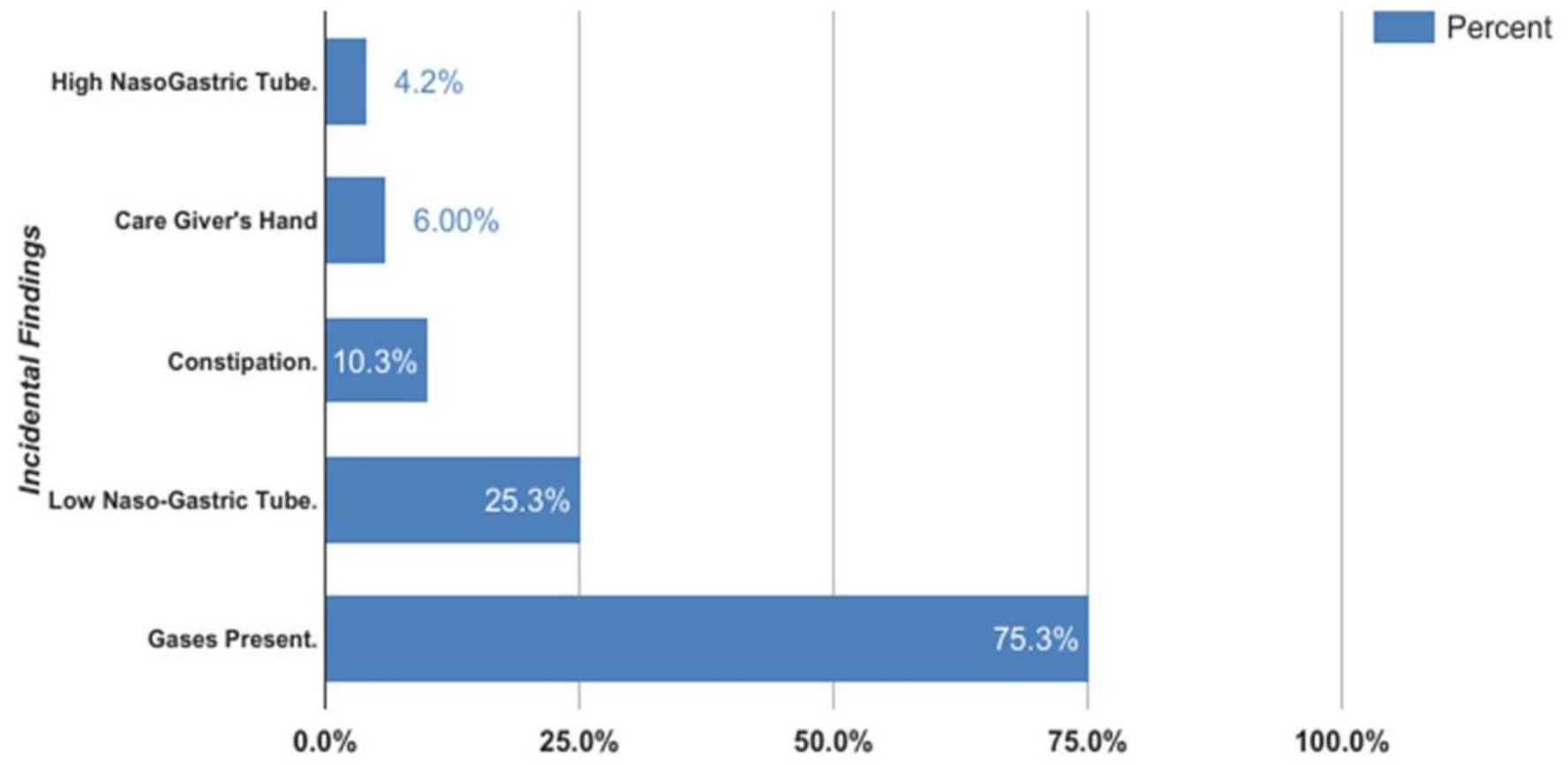

Percentage 
Figure 3

Types and percentages of incidental findings, N= 166 